\title{
The Lateral Migration of a Drop under Gravity between Two Parallel Plates at Finite Reynolds Numbers
}

\author{
A. Nourbakhsh and S. Mortazavi ${ }^{\dagger}$ \\ Mechanical Eng. Dept., Isfahan University of Technology, Isfahan, 84156-83111, Iran \\ $†$ Corresponding Author Email: saeedm@cc.iut.ac.ir
}

(Received December 13, 2009; accepted March 25, 2010)

\begin{abstract}
A finite difference / front tracking method is used to examine the lateral migration of a three-dimensional deformable drop in plane Poiseuille flow at a finite-Reynolds-number. The computations are based on an improved implementation of the front tracking method at finite Reynolds numbers that include convective terms. The elliptic pressure equation is solved by a multigrid method. Both neutrally buoyant and non-neutrally buoyant drop are studied. The computation is performed within a unit cell which is periodic in the direction along the channel. A neutrally buoyant drop lags the fluid slightly, and the wall effect balances the effect of the curvature of the velocity profile, giving rise to an equilibrium lateral position about halfway between the wall and the centerline (the SegreSilberberg effect). Results are presented over a range of density ratios. In the non-neutrally buoyant case, the gravity force is imposed along the flow direction. Non-neutrally buoyant drops have more complicated patterns of migration, depending upon the magnitude of the buoyancy force. When the density difference is small, the equilibrium position is either near the wall or near the centerline, depending on whether the drop leads or lags the local fluid. When the density difference is large enough, the equilibrium position shifts towards the centerline, irrespective of whether the drop is lighter or heavier than the fluid. The effect of Reynolds number and capillary number on the non-neutrally buoyant drops is investigated. The accuracy of the method is assessed by comparison with the other simulations and experiments.
\end{abstract}

Keywords: Neutrally and non-neutrally buoyant drop, Poiseuille flow, Density ratio, Capillary number.

NOMENCLATURE

$\begin{array}{ll}\text { a } & \text { drop radius } \\ b & \text { minimum drop dimension } \\ C a & \text { capillary number } \\ D & \text { deformation parameter } \\ \mathbf{f} & \text { body force } \\ F r & \text { Froude number } \\ \mathrm{g} & \text { acceleration of gravity } \\ H & \text { height of the channel } \\ I(\mathbf{x}) & \text { indicator function } \\ \mathrm{L} & \text { maximum drop dimension } \\ \mathbf{n} & \text { unit vector normal to the surface } \\ p & \text { pressure } \\ \operatorname{Re}_{b} & \text { bulk Reynolds number } \\ \operatorname{Re}_{d} & \text { Reynolds number based on the centerline } \\ & \text { velocity and the drop diameter } \\ \operatorname{Re}_{p} & \text { particle Reynolds number } \\ \mathbf{t} & \text { vector tangent to the edge of the element }\end{array}$

\section{INTRODUCTION}

Motion of liquid drops and cells through narrow channels and tubes has been a matter of interest for

$\begin{array}{ll}\mathbf{u} & \begin{array}{l}\text { velocity } \\ U_{c}\end{array} \\ \mathbf{x} & \begin{array}{l}\text { undisturbed channel centerline } \\ \text { velocity } \\ \text { point at which the equation is } \\ \text { evaluated }\end{array} \\ \mathbf{x}^{\prime} & \begin{array}{l}\text { Lagrangian representation of the } \\ \text { interface }\end{array} \\ \alpha & \text { density ratio } \\ \beta & \text { 2 or } 3, \text { dimension } \\ \delta & \text { smoothness parameter } \\ \kappa & \text { twice the mean curvature } \\ \lambda & \text { viscosity ratio } \\ \mu & \text { discontinuous viscosity } \\ \rho & \text { discontinuous density } \\ \sigma & \text { surface tension coefficient } \\ \tau & \text { non-dimensional time } \\ \zeta & \text { size ratio }\end{array}$

many years and there has been a considerable interest in doing research in this area. The flow of slurries, 
microfluidic systems, the recovery of oil by chemical flooding, advanced materials processing waste treatments, food processing and pharmaceutical manufacturing are typical application of these flows. In the present study, the motion of a three-dimensional neutrally and non-neutrally buoyant drop in a plane Poiseuille flow is studied by numerical simulations at finite-Reynolds-numbers. The migration of dilute suspensions of neutrally buoyant solid particles in pipe flow at a finite Reynolds number was first observed by Segre and Silberberg (1962a, b). Their experimental studies showed that the particles migrate away from both the wall and the centerline and accumulate at a certain equilibrium position of about 0.6 of the tube radius from the axis. This equilibrium position is a result of competition between a repulsion force due to the walls and a force due to the curvature of the velocity profile. This remarkable Segre-Silberberg effect has been verified by many experimental works.

For example, Goldsmith and Mason (1962) observed that a rigid particle stayed at the initial radial position at very small Reynolds numbers and migrated to an intermediate position at finite Reynolds numbers. Karnis et al. (1966) reported that neutrally buoyant particles stabilized midway between the centerline and the wall. The equilibrium position was closer to the wall for larger flow rates and closer to the center for larger particles. While experiments of Goldsmith and Mason used mostly a single drop and dilute suspensions, Kowalewski (1984) conducted experiments on the concentrated suspension of drops and showed that droplet concentration is non-uniform and has two maxima, one near the tube wall and one on the tube axis. Liu et al. (1999) presented an experimental study on the velocity of single, nearly neutrally buoyant particles in water and found that particle specific gravity has a strong effect on the particle velocity. Their experimental studies showed that the modified particle Froude number is an effective parameter to reflect the effect of density.

Several numerical methods have been used in the past to study the behavior of flow in the presence of solid boundaries. These numerical methods included volumeof-fluid, Lattice-Boltzmann (Raiskinmäki et al. 2000), finite difference, boundary-integral and finite element (Walid and Ahmed 2010) methods. Most theoretical and numerical studies constitute considerable progress towards understanding the zero- or low-Reynoldsnumber motion of drops or bubbles in channels. Chan and Leal (1979) examined the lateral migration of a Newtonian drop suspended in a parabolic flow of another Newtonian fluid and showed that for values of $\lambda$ between 0.5 and 10 ( $\lambda$ is the viscosity ratio), the drop migrates towards the walls, but for $\lambda<0.5$ and $\lambda>10$ it moves to the centerline of the channel. The creeping motion of a neutrally buoyant deformable drop flowing through a circular tube was simulated by Martinez and Udell (1990) with a boundary integral method. The effect of the capillary number, viscosity ratio and drop size on the deformation, the drop speed and the additional pressure loss was examined. The deformation of neutrally buoyant drops and bubbles moving through straight tubes and constrictions under pressure-driven flow has been studied by Tsai and Miksis (1994). They indicated that for small values of capillary number a steady-state solution can be found, but as capillary number increases large deformation for the drop is observed and the existence of a steady-state shape is not clear. Doddi and Bagchi (2008) showed that neutrally capsule deformation increases with increasing capillary number and it aligns more with the $\mathrm{x}$-axis implying that the lateral migration is essentially due to the departure from the initially spherical shape. Schleizer and Bonnecaze (1999) found that as the capillary number increases, the droplet deforms more due to the increase in viscous stresses along the interface and above critical values of the capillary number no steady shape exists. Staben et al. (2006) used a novel boundary-integral algorithm to simulate the motion of a non-neutrally buoyant sphere translating and rotating between two parallel plates in a Poiseuille flow. They indicated that the translational velocity increases with increasing particle diameter, except for particles that are so large that the upper wall has a dominant influence on the particle velocity.

Theoretical and numerical methods used in the above studies can not be applied to drop motion at finite Reynolds numbers, where inertia effects play a major role. Only a few investigators have simulated the unsteady motion of drops and particles at finite Reynolds numbers. Feng et al. (1994a,b) conducted a two-dimensional finite element simulation of the motion of a solid particle in a Poiseuille flow at finite Reynolds numbers. They observed that a neutrally buoyant particle exhibits the Segre-Silberberg effect. Mortazavi and Tryggvasson (2000) used a finitedifference/front tracking method for numerical simulation of the motion of two-dimensional neutrally buoyant drops suspended in a pressure-driven channel flow at finite Reynolds numbers. They stated that in the limit of a small Reynolds number, the motion of the drop depends strongly on the viscosity ratio. At a higher Reynolds number, the drop moves to an equilibrium position about halfway between the centerline and the wall or it undergoes oscillatory motion. Stationary bubble deformation in a steady finite Reynolds number liquid flow was simulated by Ryskin and Leal (1984) and Christov and Volkov (1985). Ryskin and Leal (1984) used a finite difference method with boundary fitted coordinate system to solve vorticity-stream function formulation of the Navier-Stokes equations for the steady rise of a deformable inviscide axisymetric bubble.

While the numerous studies mentioned above constitute considerable progress towards understanding the lowReynolds-number motion of drops and bubbles in channels, some important unresolved issues and computational challenges still remain. In particular, there is a need for a three-dimensional systematic assessment of the role of drop deformation on the migration of a deformable drop between two parallel plates at finite Reynolds numbers. In the following section, the migration of a three-dimensional neutrally and non-neutrally buoyant drop is studied by employing a finite difference / front tracking method. The present study is similar to that predicted by Feng et al. (1994b), except that the particles are deformable and threedimensional. 
The paper is organized as follows: the governing equations and numerical method are discussed in section 2. The results of several three-dimensional simulations are reported in section 3. In section 3.2, the lateral migration of a neutrally buoyant drop is investigated. In section 3.3, the migration of a nonneutrally buoyant drop is simulated. The effect of density ratio, capillary number and Reynolds number is addressed, in this part. Section 4 contains the conclusion.

\section{GOVERNING EQUATIONS AND NUMERICAL METHOD}

\subsection{Problem Setup}

The geometry of the flow is shown in Fig. 1. The motion of a drop is studied in a channel that is bounded by two flat plates in the z-direction. The height and length of the channel are $H$. To decrease the computation time, the depth of the channel in the $y$ direction is taken to be $0.5 \mathrm{H}$. In absence of the drop, the undisturbed flow is a fully-developed parabolic (Poiseuille) flow and is driven by a constant pressure gradient. In the problem formulation (Mortazavi and Tryggvason 2000):

$\nabla p=\nabla p_{o}+\nabla p^{\prime}$

where $\nabla p_{o}$ is the externally specified pressure gradient and $\nabla p^{\prime}$ is the perturbation pressure gradient to be computed as part of the solution.

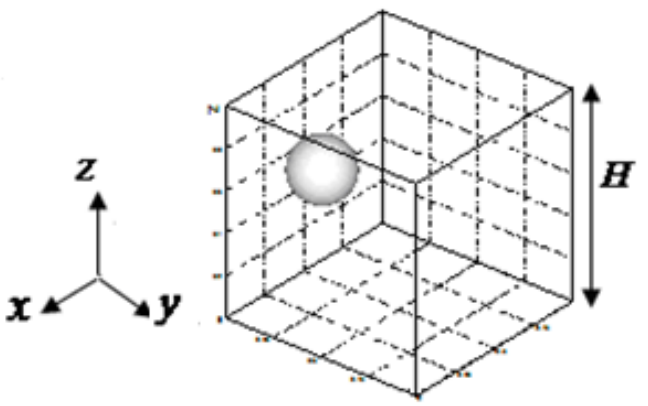

Fig. 1. The geometry for the simulation of a drop in a channel

\subsection{Boundary Conditions}

The boundary condition on the plates is the no-slip condition. The domain is periodic in the $\mathrm{x}-$ and $\mathrm{y}$ directions. Normal stresses show the jump across the interface by surface tension and tangential stresses are continuous on the surface of the drop.

\subsection{Dimensionless Parameters}

The governing non-dimensional numbers are as follows: the ratio of the viscosity of the drop fluid to the suspending medium $\lambda=\mu_{i} / \mu_{o}$, the density ratio $\alpha=\rho_{i} / \rho_{o}$, and the ratio of the radius of the drop to the height of the channel $\zeta=a / H$. The viscosity and density of the drop liquid are denoted by $\mu_{i}$ and $\rho_{i}$, respectively, and the suspending fluid has viscosity $\mu_{o}$ and density $\rho_{o}$. The bulk Reynolds number is defined in terms of the undisturbed channel centerline velocity $\left(U_{c}\right)$ and the channel height, as $\operatorname{Re}_{b}=\rho_{o} U_{c} H / \mu_{o}$. A Reynolds number based on the centerline velocity and the drop diameter (d) is defined by $\operatorname{Re}_{d}=\rho_{o} U_{c} d / \mu_{o}$. A particle Reynolds number can be defined as $\operatorname{Re}_{p}=\rho U_{c} a^{2} / \mu H$. The capillary number, $C a=U_{c} \mu_{o} / \sigma$ describes the ratio of the viscous stress to the interfacial tension. The Froude number is defined as $F r=g L / U_{c}^{2}$. Non-dimensional time is defined by $\tau=t U_{c} / H$.

\subsection{Numerical Method}

One of the important topics that has been considered by fluid mechanics researchers is flows with interfaces. Different numerical methods are used and developed for simulating these flows. These methods can be divided into two groups, depending on the type of grids used: moving grid and fixed grid. Two important approaches of fixed-grid methods, namely the volumeof-fluid (VOF) and level-set approaches, are among the most commonly used methods. The volume-of-fluid method uses a marker function. The main difficulty in using VOF method has been the maintenance of a sharp boundary between the different fluids and the computation of the surface tension. The level-set method defines the interface by a level-set function $\phi$, but this approach has some difficulties in preserving the mass conservation.

There are recent efforts in conserving mass in level-set method (see for example Gorokhovski and Herrmann 2008). Another method presented in this paper is the finite difference /front tracking method which improved the disadvantages of the previous methods. This approach was described in detail by Unverdi and Tryggvason (1992a, b) and only a brief outline is given here. The present computations are based on an improved implementation of the front tracking method at finite Reynolds numbers that include convective terms. The numerical technique is based on a direct discretization of the Navier-Stokes equation. In conservative form it is

$$
\begin{aligned}
& \frac{\partial \rho \mathbf{u}}{\partial t}+\nabla \cdot(\rho \mathbf{u u})=-\nabla p+\rho \mathbf{f}+\nabla \cdot \mu\left(\nabla \mathbf{u}+\nabla^{T} \mathbf{u}\right) \\
& +\int \sigma \kappa^{\prime} \mathbf{n}^{\prime} \delta^{\beta}\left(\mathbf{x}-\mathbf{x}^{\prime}\right) d s^{\prime}
\end{aligned}
$$

where, $\mathbf{u}$ is the velocity, $p$ is the pressure, and $\rho$ and $\mu$ are the discontinuous density and viscosity fields, respectively. $\sigma$ is the surface tension coefficient, $\mathbf{f}$ is a body force, and surface forces are added at the interface. The term $\delta^{\beta}$ is a two- or three-dimensional $\delta$ function constructed by repeated multiplication of one-dimensional $\delta$ functions. The dimension is denoted by $\beta=2$ or $3, \kappa$ is the curvature for two-dimensional flow and twice the mean curvature for threedimensional flows, $\mathbf{n}$ is a unit vector normal to the front, $\mathbf{x}$ is the point at which the equation is evaluated, and $\mathbf{X}^{\prime}$ is a Lagrangian representation of the interface. 
This equation is solved by a second-order projection method using centred differences on a fixed regular, staggered grid. Both the drop and the ambient fluid are taken to be incompressible, so the velocity field is divergence free (Tryggvason et al. 2001):

$$
\nabla \cdot \mathbf{u}=0
$$

Equation (3), when combined with the momentum equation, leads to a non-separable elliptic equation for the pressure. If the density is constant, the elliptic pressure equation is solved by fast Poisson solver (FISHPACK), but when the density of the drop is different from the suspending fluid, the equation is solved by a multigrid method (Adams 1989).

Equations of state for the density and the viscosity are:

$$
\frac{D \rho}{D t}=0, \quad \frac{D \mu}{D t}=0
$$

here, $D / D t$ is the material derivative, and Eq. (4) simply states that the density and the viscosity of each fluid remain constant. As the drops move and deform, the density and the viscosity need to be updated. This is done by solving a Poisson equation for an indicator function $I(\mathbf{x})$ such that (Unverdi and Tryggvason 1992b):

$$
\begin{aligned}
& \rho(\mathbf{x})=\rho_{o}+\left(\rho_{i}-\rho_{o}\right) I(\mathbf{x}) \\
& \mu(\mathbf{x})=\mu_{o}+\left(\mu_{i}-\mu_{o}\right) I(\mathbf{x})
\end{aligned}
$$

An indicator function that is 1 inside the drop and 0 in the outer fluid is constructed from the known position of the interface. In two-dimensional flow:

$$
I(\mathrm{x}, \mathrm{y})=\frac{1}{2 \pi} \oint \frac{\mathrm{r} \cdot \mathbf{n}}{\mathrm{r}^{2}} g(r) d s
$$

where $g(r)=1-e^{-r^{2} / \delta^{2}}$, and $\delta$ is a smoothness parameter.

The singularities at the front (density and viscosity gradients and surface tension) are approximated on the fixed grid by smooth functions with a compact support. The density and viscosity fields are reconstructed at each time step by integrating the smooth grid delta function, after the front has been moved, and the body force due to surface tension is added to the nodal values of the discrete Navier-Stokes equations.

\subsection{The Structure of the Front}

In the present method, the governing equations are solved on a fixed Eulerian grid, and the interface is tracked in a Lagrangian manner by a set of marker points (front) (Fig. 2). The front is represented by separate computational points that are moved by interpolating their velocities from the grid. These points are connected by triangular elements to form a front that is used to keep the density and viscosity stratification sharp and to calculate surface tension. At each time step, information must be passed between the front and the stationary grid. This is done by a method similar to the one discussed by Unverdi and Tryggvason (1992a), which spreads the density jump to the grid points next to the front and generates a smooth density field that changes from one density to the other over two to three grid spaces. As the front moves, it deforms and stretches. The resolution of some parts of the front becomes inadequate, while other parts contain large number of elements.

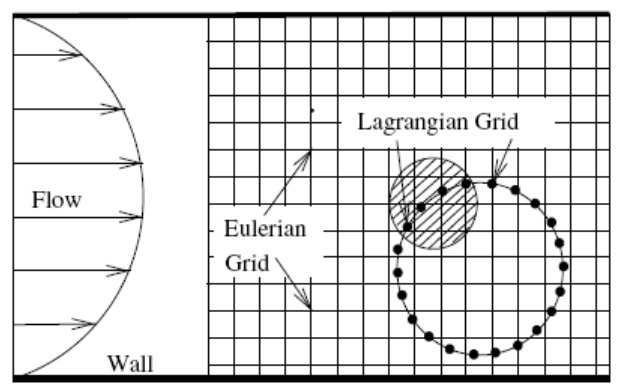

Fig. 2. The Eulerian and Lagrangian grids. From Doddi and Bagchi (2008), with permission.

To maintain accuracy, either additional elements must be added when the separation of points becomes too large or the points should be redistributed to maintain adequate resolution. Also, it is generally desirable to remove small elements. In addition to reducing the total number of elements used to represent the front, element removal usually prevents the formation of 'wiggles' much smaller than the grid size.

\subsection{Computing Surface Tension}

The surface tension force is computed from the geometry of the interface and distributed to the fixed grid. Generally, curvature is very sensitive to minor irregularity in the interface shape, and it is difficult to achieve accuracy and robustness at the same time. However, the surface tension force is computed on each surface element by (Tryggvason et al. 2001):

$$
\begin{aligned}
& \delta \mathbf{F}_{\sigma}=\sigma \int_{\delta A} \mathbf{n} d A=\sigma \int_{\delta A}(\mathbf{n} \times \nabla) \times \mathbf{n} d A \\
& =\sigma \oint_{s} \mathbf{t} \times \mathbf{n} d s
\end{aligned}
$$

where the integration is over the boundary curve of each element. The cross product is a vector that lies on the surface and is normal to the edge of the element. The product of the surface-tension and this vector gives the 'pull' on the edge and the net pull is obtained by integrating around the perimeter of the element. If the element is flat, the net force is zero, but if the element is curved, the net force is normal to it when the surface tension is constant

\section{RESUlTS AND DisCUSSION}

\subsection{Resolution Test}

To ensure grid independence of the solution, resolution tests were performed at three different grid resolutions with $32 \times 16 \times 32, \quad 64 \times 32 \times 64$ and $128 \times 64 \times 128$ grid points. The size of the computational domain is $1 \times 0.5 \times 1$. The sensitivity of the results to mesh resolution is examined in Fig. 3, which shows the trajectory and axial velocity for a three dimensional drop at $\operatorname{Re}_{d}=10$. The other flow parameters are: $C a=0.05, \lambda=1, \alpha=4$ and $\zeta=0.125$. There is no 
gravity force in these simulations. The drop is initially released close to the upper wall $(z / H=0.825)$ and migrates to an equilibrium lateral position about halfway between the wall and the centerline (the SegreSilberberg effect). The difference between the results predicted using $64 \times 32 \times 64$ and $128 \times 64 \times 128$ grids is much smaller than the difference between the results predicted using $32 \times 16 \times 32$ and $64 \times 32 \times 64$ grids. As a result, the solution converges with grid refinement, and grid-independent results are reached as the mesh is refined. The simulation for a $64 \times 32 \times 64$ grid took approximately $2500 \mathrm{~min}$ to reach $\tau=60.2057$, using an AMD Opteron(tm) Dual-Core 2216 Processor under Visual Fortran 6.
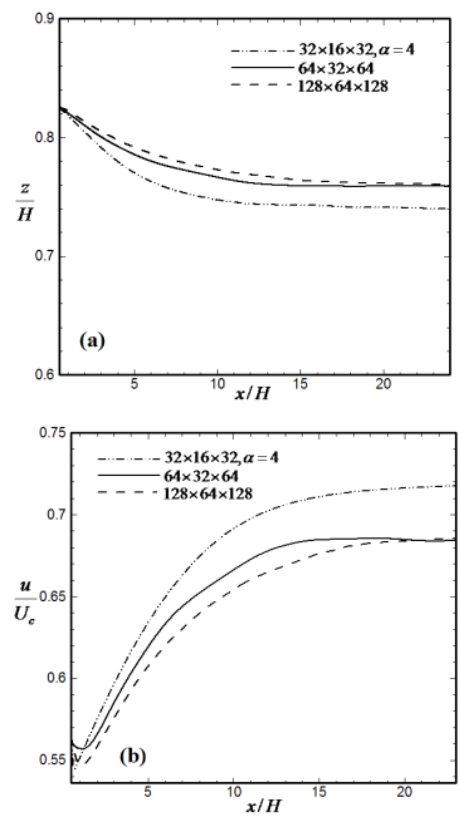

Fig. 3. Effect of resolution on the lateral migration of a drop in a plane Poiseuille flow. The lateral position and the axial velocity versus the axial location of a drop at three different grid resolutions. The flow parameters are: $\operatorname{Re}_{d}=10, C a=0.05, \lambda=1, \alpha=4$ and $\zeta=0.125$.

The streamlines at steady-state are plotted in Fig. 4 for a drop with $64 \times 32 \times 64$ grid points in a plane through the center of the drop $(z=0.25 H)$ after the drop reaches an equilibrium lateral position. We next present the results of simulations on neutrally and non-neutrally buoyant drops migrating in Poiseuille flow and compare them with available simulations and experiments.

\subsection{Neutrally Buoyant Drops}

In a recent paper, the effects of capillary number, Reynolds number, viscosity ratio and drop size on the migration of a neutrally buoyant drop were investigated (Nourbakhsh and Mortazavi 2010). In the current effort, numerical studies are extended to investigate the effect of the density ratio.

\subsubsection{Effect of the Density Ratio}

Figure 5 shows the lateral positions versus the axial location for a drop that is released at different initial positions. The flow parameters are: $\operatorname{Re}_{d}=10$, $C a=0.05, \quad \lambda=1, \quad \alpha=4$ and $\zeta=0.125$. The grid resolution is $64 \times 32 \times 64$ in the $\mathrm{x}-, \mathrm{y}$ - and z-directions, respectively.

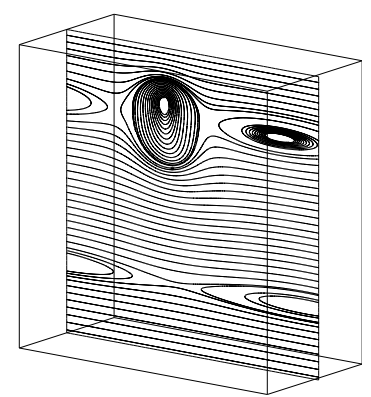

Fig. 4. Steady-state streamlines for a drop with a $64 \times 32 \times 64$ grid. The flow parameters are:

$\operatorname{Re}_{d}=10, C a=0.05, \lambda=1, \alpha=4$ and $\zeta=0.125$.

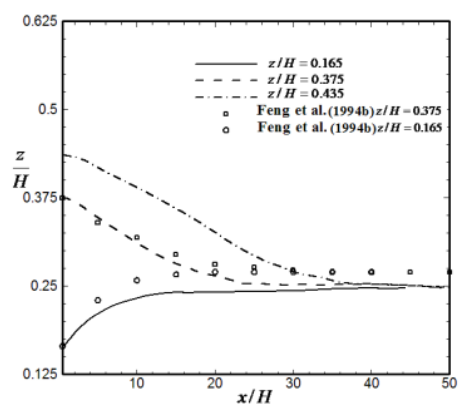

Fig. 5. Comparison of the simulated Segre-Silberberg effect with the simulation of Feng et al. (1994b). The drop released at $z=0.165 \mathrm{H}, z=0.375 \mathrm{H}, z=0.435 \mathrm{H}$, $\alpha=4$ and $\operatorname{Re}_{d}=10$ in the present study and released at $z=0.165 H$ and $z=0.375 H$ with $\operatorname{Re}_{p}=0.625, \operatorname{Re}_{b}=40$ in simulation of Feng et al. (1994b).

All drops with different initial positions move to an equilibrium lateral position about halfway between the centerline and the wall according to the so-called SegreSilberberg effect (Segre and Silberberg 1962b; Oliver 1962; Ho and Leal 1974). For deformable drops, the wall repulsion increases and this effect moves the equilibrium lateral position closer to the centerline of the channel. The lateral position is compared with the simulation of Feng et al. (1994b) in Fig. 5 at initial positions: $z / H=0.165$ and $z / H=0.375 .(z / H$ is the dimensionless lateral position). The equilibrium position is close to that predicted by Feng et al. (1994b) and difference is due to the fact that these researchers studied a rigid sphere, while we considered a deformable particle. (The drop in Fig. 5 has been released in the lower half of the computational domain and the equilibrium distance from the lower wall is $\left.z_{e q} / H=0.25\right)$.

The effect of the density ratio on the lateral migration of a drop was examined by carrying out four simulations with $\alpha=1,2,4$ and 8 . The other flow parameters are: $\operatorname{Re}_{d}=10, \quad C a=0.05, \quad \lambda=1$ and $\zeta=0.125$. Figure 6 a shows the lateral position versus 
its axial location. It is clear that the drops move to an equilibrium position closer to the wall with increasing the density ratio in agreement with simulations reported by Mortazavi and Tryggvason (2000). (The drop in Fig. 6a has been released in the upper half of the computational domain and the equilibrium distance from the upper wall is $z_{e q} / H=0.25$ for $\alpha=2$ ).

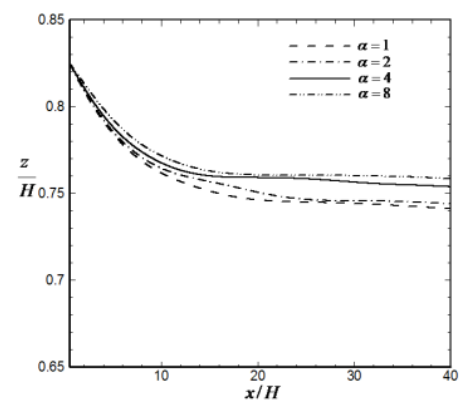

(a)

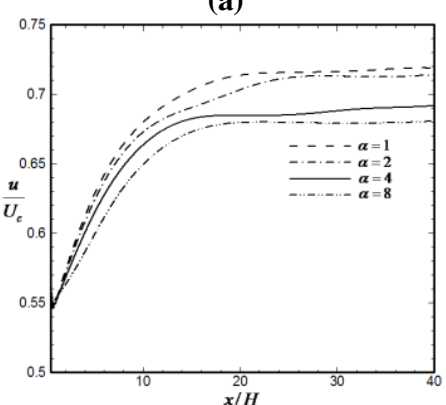

(b)

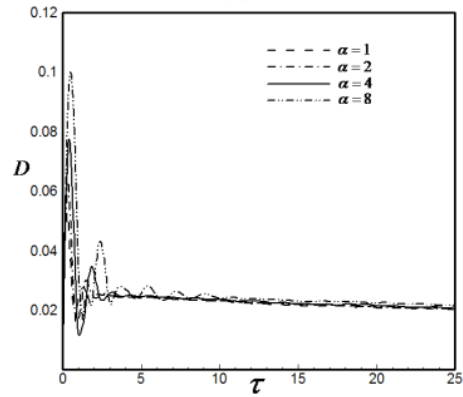

(c)

Fig. 6. (a)The lateral position, (b) axial velocity versus the axial location and (c) drop deformation versus nondimensional time at $\operatorname{Re}_{d}=10$.

The axial velocities of the drops are plotted versus the axial location in Fig. 6b. The axial velocity increases with decreasing density ratio. The drop with smaller density ratio migrates to a lower equilibrium position, and therefore, its axial velocity is larger. The deformation of the drop is examined by considering the Taylor deformation parameter defined by $D=(L-b) /(L+b)$ (Taylor 1934). The deformation of the drops is plotted versus time in Fig. 6c. The drops start deforming from a spherical shape, and then reach a steady state shape.

The rate of deformation is significant during the initial transient period. It is clear from Fig. $6 \mathrm{c}$ that the deformation increases slightly as the density ratio increases during the initial transient period and is nearly the same at steady state.

\subsection{Non-Neutrally Buoyant Drops}

In this section, the motion of a non-neutrally buoyant drop is studied, and the effects of density ratio, capillary number and Reynolds number are investigated.

\subsubsection{Effect of the Density Ratio}

Numerical simulations of the motion of drops with various densities have been performed. The gravity force is imposed along the flow direction. The lateral position of the drop is plotted versus the axial location in Fig. 7a. The flow parameters are: $\operatorname{Re}_{d}=10, C a=0.1$, $F r=30, \lambda=1$ and $\zeta=0.125$. The calculations were done on a $64 \times 32 \times 64$ grid. The simulation for $\alpha=0.8$ took approximately $2850 \mathrm{~min}$ to reach $\tau=60.2057$, using an AMD Opteron(tm) Dual-Core 2216 Processor under Visual Fortran 6.

These trajectories can be interpreted based on the understanding of the migration of a buoyant drop and the effect of the curvature of the velocity profile. For neutrally buoyant drops, the flow through the gap between the drop and the wall leads to a repulsive lubrication force called 'geometric blocking' by Feng et al. (1994b), that pushes the drop away from the wall. The negative slip velocity and the curvature of the velocity profile generate a force that drives the drop away from the center of the channel. So, these two forces move the drop to an equilibrium position about halfway between the centerline and the wall.

In the non-neutrally buoyant case, the buoyancy force generates a slip velocity. If the drop is heavier than the ambient fluid $(\alpha>1)$ and the density difference is small, the slip velocity is larger than a neutrally buoyant drop (the lagging velocity is larger), resulting a larger inertial lift that moves the drop somewhere close to the centerline $(\alpha=1.01)$. Our numerical simulatio ns predict the same behavior as observed by Feng et al. (1994b) qualitatively. The difference can be attributed to the fact that Feng et al. (1994b) studied two-dimensional rigid spheres, while we considered three-dimensional deformable particles. The equilibrium position predicted here $\left(z_{e q} / H \approx 0.293\right)$ is closer to the wall than that predicted by Feng et al. (1994b) $\left(z_{\text {eq }} / H \approx 0.35\right)$. Experimental studies observed by Jeffery and Pearson (1965) also showed that when the particles were slightly denser than the fluid, they migrated relatively rapidly to the wall of the tube for downward fluid flow and to the axis of the tube for upward fluid flow. The present result is also similar to that predicted by Oliver (1962).

When the density difference is large enough, the lag velocity is larger, the inertial lift and wall effect are dominant so the drop will be very close to the centerline $(\alpha=1.1)$. This result is compared with that predicted by Feng et al. (1994b) at $\alpha=1.1$ in Fig. 7a. The equilibrium position is similar to the result of Feng et al. (1994b). Denson et al. (1966) also investigated particle migration in shear fields at finite Reynolds numbers. They found that a sphere lags behind the fluid and the slip velocity is negative, 
thereby giving rise to a transverse force directed towards the axis. Karnis et al. (1963) also studied the motion of spheres that were denser than the entraining liquid and observed outward migration to a radial position of 0.5 .

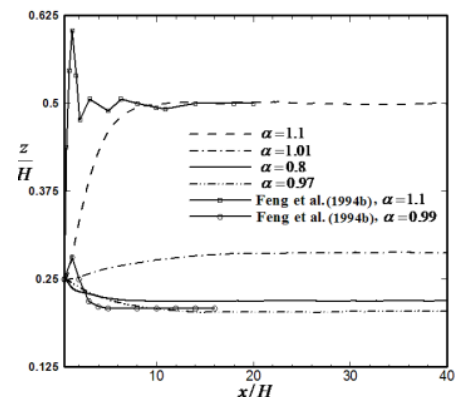

(a)

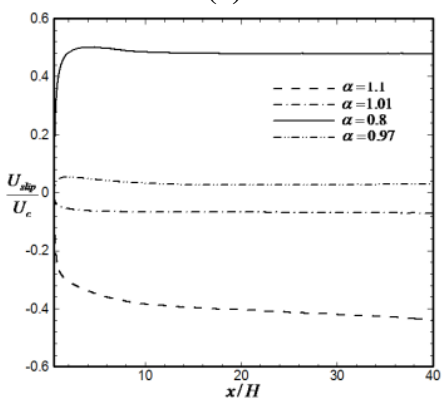

(b)

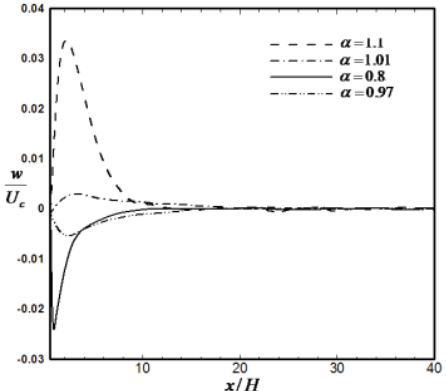

(c)

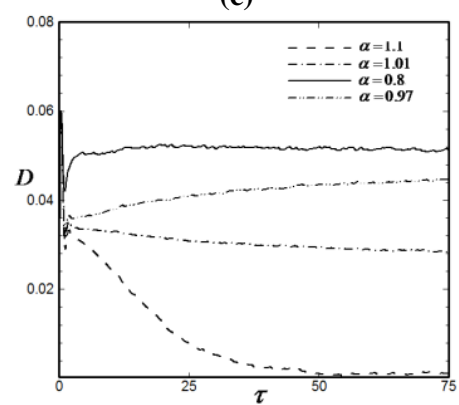

(d)

Fig. 7a. Comparison of the lateral position with the simulation of Feng et al. (1994b). The parameters are:

Feng et al.: $\operatorname{Re}_{d}=30$ and $F r=43.56$; the present simulation: $\operatorname{Re}_{d}=10$ and $F r=30$, (b) slip velocity, (c) lateral velocity versus the axial position and (d) drop deformation versus non-dimensional time at four different density ratios.

If the drop is lighter than the ambient fluid and the density difference is small, the slip velocity will be positive. The curvature of the velocity profile moves the drop closer to the wall $(\alpha=0.97)$. The equilibrium position predicted here $\left(z_{e q} / H \approx 0.2\right)$ is close to that predicted by Feng et al. (1994b) for $\alpha=0.99$ ( $z_{e q} / H \approx 0.208333$ ) (Fig. 7a).

If the density difference is sufficiently large and the drop is lighter than the fluid, the motion of drop is dominated by the slip velocity. The wall lubrication force also increases significantly. The inertial lift, however, increases as well, but to a lower extend, and the equilibrium position gets closer to the centerline ( $\alpha=0.8)$. Again, the equilibrium position is close to the result of Feng et al. (1994b). The equilibrium position predicted here $\left(z_{e q} / H \approx 0.225\right)$ is closer to the wall than that predicted by Feng et al. (1994b) for $\alpha=0.85\left(z_{e q} / H \approx 0.291\right)$.

Theory of Vasseur and Cox (1976) has some interesting results that can be compared to our simulations. In the non-neutrally buoyant case, they found that:

(a) When the drop is very slightly buoyant, it moves either the wall or the centerline depending on whether it leads or lags the fluid.

(b) When the drop is slightly buoyant, it moves to an equilibrium position between the centerline and the wall, and is close to the centerline if the drop lags but close to a wall if the drop leads the fluid. This result is similar to our simulations ( $\alpha=1.01$ and 0.97 ).

(c) The equilibrium position of a drop always approaches the centerline as the slip velocity increases, whether leading or lagging. This can be compared to our simulations with $\alpha=0.8$ and 1.1. This result is similar to the current work for a lagging drop $(\alpha=1.1)$. But, in our case, the equilibrium position moves closer to the centerline as the slip velocity increases for a leading drop. This reversal behavior does not agree with theory of Vasseur and Cox (1976), but is in agreement with numerical simulations of Feng et al. (1994b).

The slip velocity is defined as the axial velocity of the drop minus the undisturbed fluid velocity at the drop center. The slip velocities are plotted versus the axial location in Fig. 7b. If the drop is lighter than the fluid ( $\alpha=0.97)$, the slip velocity is positive and if the drop is heavier than the fluid $(\alpha=1.01)$, the slip velocity is negative, which is consistent with that reported by Denson et al. (1966). It is clear from Fig. 7b that when the density difference is sufficiently large, the slip velocity also gets larger $(\alpha=0.8,1.1)$ (Feng et al. 1994b).

The lateral velocity of the drop is plotted versus the axial location in Fig. 7c. The lateral velocity becomes larger during the initial period. When the drop reaches to an equilibrium lateral position, the lateral velocity becomes zero. The lateral velocity of the heavier drops is positive and the lateral velocity of the lighter drops is negative. We note that slight difference in density between drop and the ambient fluid lead to larger 
migrating velocities than neutrally buoyant drops that was stated by Nourbakhsh and Mortazavi (2010).

The deformation of the drops is plotted versus time in Fig. $7 \mathrm{~d}$. The difference between deformation of drops that are lighter than the ambient fluid is very little (less than 0.01). The heaviest drop $(\alpha=1.1)$ is similar to a rigid sphere and its deformation is small.

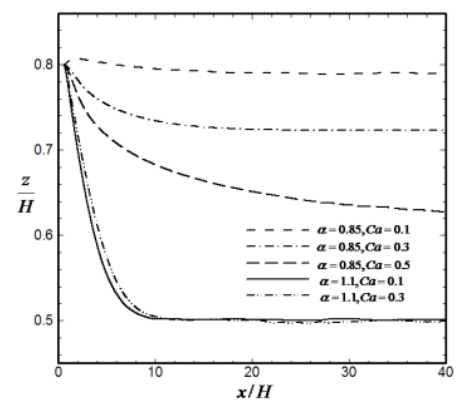

(a)

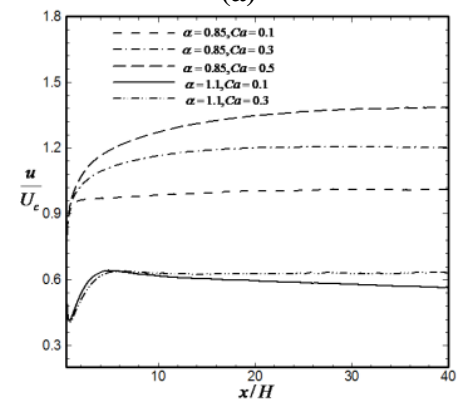

(b)

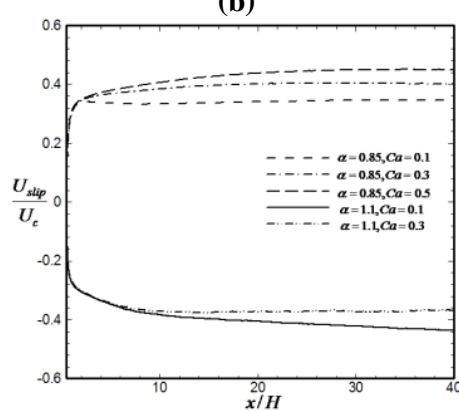

(c)

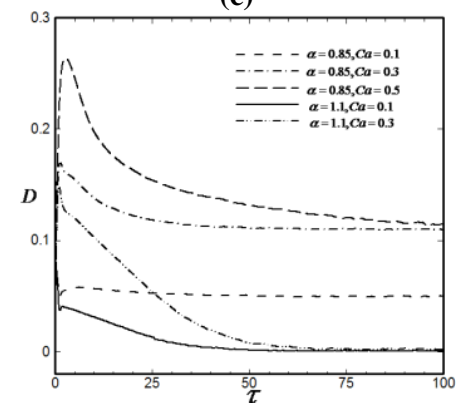

(d)

Fig. 8. (a)The lateral position, (b) axial velocity, (c) slip velocity versus the axial position and (d) drop deformation versus non-dimensional time at $\operatorname{Re}_{d}=10$.

\subsubsection{Effect of the Capillary Number}

To illustrate the role of deformation at a finite Reynolds number, the motion of drops with various capillary numbers is investigated. In Fig. 8a, the lateral position of the drop is plotted versus the axial location. Three simulations were performed at $\alpha=0.85, \quad \mathrm{Ca}=0.1$, 0.3 and 0.5 ; and two simulation were performed at $\alpha=1.1, C a=0.1$ and 0.3 . The other flow conditions are: $\operatorname{Re}_{d}=10, F r=30, \lambda=1$ and $\zeta=0.125$.

It is clear that the drop migrates faster towards the channel centre with increasing capillary number. Similar behavior has been indicated by Griggs et al. (2007), Janssen and Anderson (2008) and Doddi and Bagchi (2008) for the neutrally buoyant drop migration in the limit of creeping flow. Since the inertial lift and wall effect are dominant, the heavier drop $(\alpha=1.1)$ migrates to the centerline, irrespective of the value of capillary number.

The axial velocities of the drops are plotted versus the axial location in Fig. 8b. For both density ratios ( $\alpha=0.85,1.1$ ), the axial velocity increases with increasing capillary number. Griggs et al. (2007) and Nourbakhsh and Mortazavi (2010) also showed that the axial velocity is an increasing function of capillary number. Martinez and Udell (1990) reported that the axial velocity of a neutrally buoyant drop increases with increasing capillary number due to larger drop deformation.

The slip velocities are plotted versus the axial location in Fig. 8c. The slip velocity of the lighter drop is positive and the slip velocity of the heavier drop is negative, which is consistent with the findings of Denson et al. (1966). As the capillary number increases, the slip velocity of the heavier drop decreases in agreement with the result of Mortazavi and Tryggvason (2000), but the slip velocity of the lighter drop increases. In this case, the magnitude of the slip velocity is larger than in the case of a neutrally buoyant drop (see Nourbakhsh and Mortazavi 2010). This is consistent with that reported by Jeffrey and Pearson (1965).

The deformation of drops is plotted versus time in Fig. 8d. It is clear that the drop deformation increases with increasing capillary number due to the increase in viscous stresses along the interface. However, the deformation of the heavier drop is nearly zero $(\alpha=1.1)$.

Figure 9 shows the streamlines for drops with $\mathrm{Ca}=0.1$ and $0.3(\alpha=0.85)$. The flow patterns are similar. When the capillary number is small, the drop maintains a nearly spherical shape. As the capillary number increases, the drop elongates. This result is in agreement with that reported by Griggs et al. (2007), Janssen and Anderson (2008), Tsai and Miksis (1994), Doddi and Bagchi (2008) and Schleizer and Bonnecaze (1999) who indicated that the drop deformation increases with increasing capillary number. Kang et al. (2005) also studied the displacement of a threedimensional droplet subject to gravitational forces and observed that the droplet is stretched larger as the capillary number becomes larger.

These computations show that at small values of capillary number, the drop deforms and attains a stationary shape. However, there exists a critical capillary number beyond which the drop is not stable to 
the imposed shear field. Based on numerical experiments that were performed, we found that steady state drop shapes are obtained up to $\mathrm{Ca}=0.8$ for $\alpha=0.85$ and $C a=1.1$ for $\alpha=1.1$. Above these critical values of capillary number, the drop does not obtain a steady shape but instead, continuously deforms and eventually breaks up.

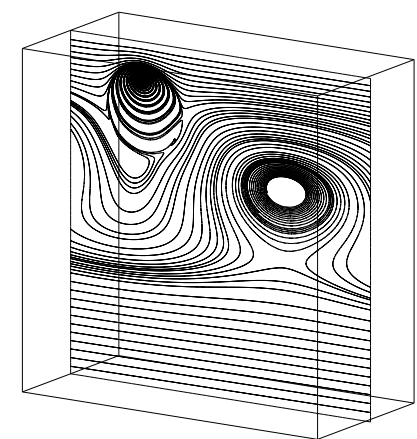

(a)

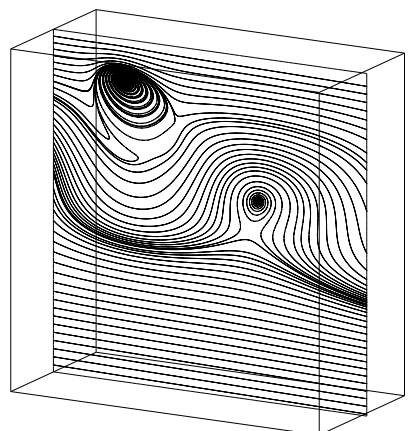

(b)

Fig. 9. Streamlines for a drop at $\operatorname{Re}_{d}=10, F r=30$, $\lambda=1, \alpha=0.85$ and $\zeta=0.125$. The capillary number is 0.1 for (a) and 0.3 for (b).

\subsubsection{Effect of the Reynolds Number}

Figure 10a shows the lateral position of the drop versus the axial location at two Reynolds numbers $(5,10)$. The simulations were performed with two density ratios: $\alpha=0.85$ and 1.1. The other flow parameters are: $C a=0.3, F r=30, \lambda=1$ and $\zeta=0.125$. The drop moves to an equilibrium lateral position. As the Reynolds number increases, the equilibrium position of lighter drops moves slightly closer to the wall. The result is in agreement with the theory of Schonberg and Hinch (1989), who found that the equilibrium position of a neutrally buoyant sphere moves towards the wall as the Reynolds number increases. Yang et al. (2005), Asmolov (1999), Mortazavi and Tryggvason (2000) and Nourbakhsh and Mortazavi (2010) also indicated that the equilibrium position of a neutrally buoyant drop moves towards the wall with increasing Reynolds number. As the Reynolds number increases, the equilibrium position of the heavier drops moves towards the centerline.

In Fig. 10b, the slip velocities of the drops are shown versus the axial location. For both density ratios, the magnitude of slip velocities increases with increasing Reynolds number in agreement with the predictions of
Yang et al. (2005). Figure 10c shows slight increase of the deformation of the lighter drop (less than 0.025) and slight decrease of the heavier drop deformation $(\approx 0.05)$ with increasing Reynolds number. The average axial velocity in the channel is plotted versus the lateral position in Fig. 11 at two Reynolds numbers. Since the shear rate increases slightly with increasing Reynolds number (Fig. 11a), the drop deformation is slightly larger at higher Reynolds number. In the case of heavier drop, the shear rate decreases slightly with increasing Reynolds number (Fig. 11b). So, the deformation of a heavier drop decreases with increasing Reynolds number.

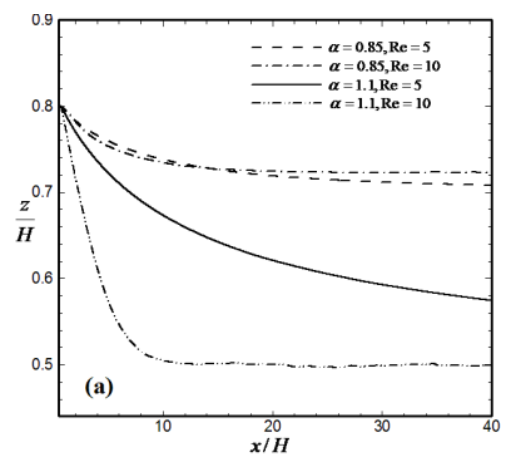

(a)

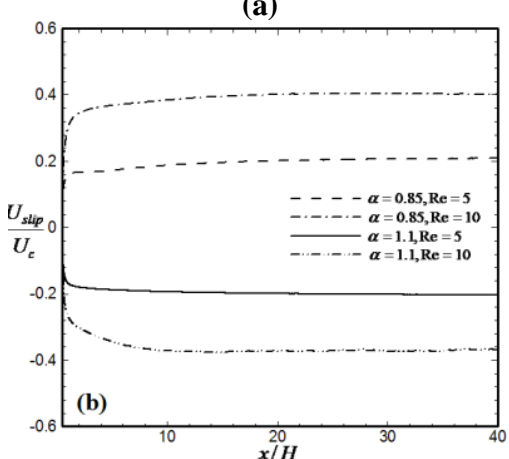

(b)

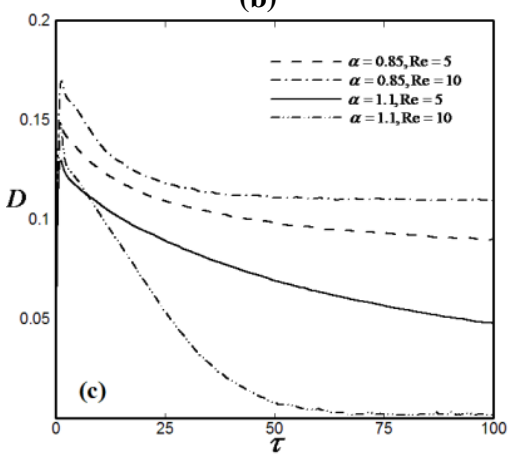

(c)

Fig. 10. (a) The lateral position, (b) slip velocity versus the axial position and (c) drop deformation versus nondimensional time at different Reynolds numbers.

\section{Conclusion}

In the present study, a finite difference / front tracking method has been used to simulate the motion of a neutrally and non-neutrally buoyant drop in plane Poiseuille flow at finite Reynolds numbers. The effect of the density ratio on the equilibrium position was 
studied for a neutrally buoyant drop. The equilibrium position moves slightly towards the wall as the density ratio increases. The drop deformation is nearly the same at steady state for drops with different densities.

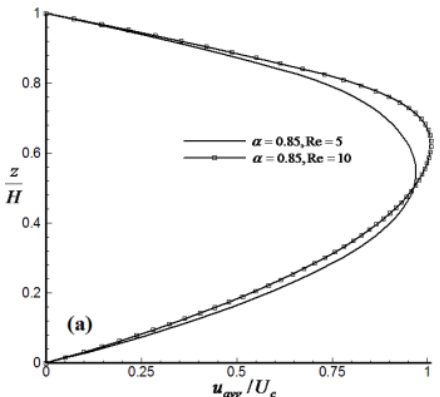

(a)

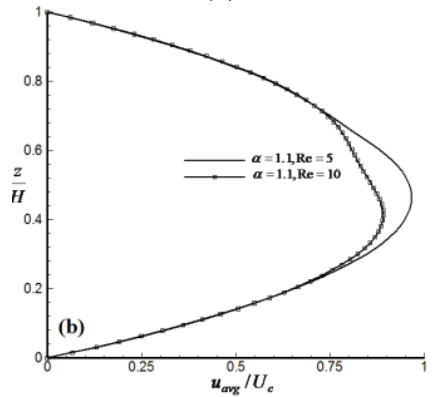

(b)

Fig. 11. The average axial velocity versus the lateral position at $\operatorname{Re}=5,10$. The density ratio is 0.85 for (a) and 1.1 for (b)

The motion of a non-neutrally buoyant drop was also studied and the effects of various non-dimensional parameters were examined. A drop that is slightly buoyant stabilizes at a position close to the wall or the centerline, depending on whether it leads or lags the undisturbed fluid. When the density difference is large enough, the equilibrium position of a lagging drop approaches the centerline. The equilibrium position of a leading drop starts to move back to the center as the slip velocity increases. If the drop is lighter than the ambient fluid, the slip velocity is positive and if the drop is heavier than the ambient fluid, the slip velocity is negative. When the density difference is sufficiently large, the slip velocity gets even larger. Slight difference in density between drop and the ambient fluid lead to larger migrating velocities than neutrally buoyant drops. The drop deformation decreases slightly with increasing the density ratio.

Effects of capillary number and Reynolds number were also studied for a non-neutrally buoyant drop. Increasing the capillary number moves the equilibrium position closer to the centerline. As the capillary number increases, the slip velocity of a heavier drop decreases, but the slip velocity of a lighter drop increases. The slip velocities were larger for nonneutrally buoyant drops compared to neutrally buoyant drops. The drop deformation increases with increasing capillary number. However, the deformation of heavier drops is small. As the Reynolds number increases, the equilibrium position of lighter drops moves slightly closer to the wall, but the equilibrium position of the heavier drops moves towards the centerline. The slip velocities of lighter and heavier drops increase with increasing Reynolds number. As the Reynolds number increases, the deformation increases slightly for a lighter drop and decreases slightly for a heavier drop.

\section{REFERENCES}

Adams, J. (1989). MUDPACK: Multigrid FORTRAN software for the efficient solution of linear elliptic partial differential equations. Appl. Math. Comput. 34, 113-146.

Asmolov, E.S. (1999). The inertial lift on a spherical particle in a plane Poiseuille flow at large channel Reynolds number. J. Fluid Mech. 381, 63-87.

Chan, P.C. and L.G. Leal (1979). The motion of a deformable drop in a second-order fluid. J. Fluid Mech. 92, 131-170.

Christov, C.I. and P.K. Volkov (1985). Numerical investigation of the steady viscous flow past a stationary deformable bubble. J. Fluid Mech. 158, 341-364.

Denson, C.D., E.B. Christiansen and D.L. Salt (1966). Particle migration in shear fields. AIChE. J. 12, 589.

Doddi, S.K. and P. Bagchi (2008). Lateral migration of a capsule in a plane Poiseuille flow in a channel. Int. J. Multiphase Flow 34, 966-986.

Feng, D.J., H.H. Hu and D.D. Joseph (1994a). Direct simulation of initial value problems for the motion of solid bodies in a Newtonian fluid: Part1. Sedimentation. J. Fluid Mech. 216, 95-134.

Feng, D.J., H.H. Hu and D.D. Joseph (1994b). Direct simulation of initial value problems for the motion of solid bodies in a Newtonian fluid: Part2. Couette and Poiseuille flows. J. Fluid Mech. 277, 271-301.

Goldsmith, H.L. and S.G. Mason (1962). The flow of suspensions through tubes. I. Single spheres, rods and discs. J. Colloid Sci. 17, 448-476.

Gorokhovski, M. and M. Herrmann (2008). Modeling primary atomization. Annual Review of Fluid Mech 40, 343-366.

Griggs, A.J., A.Z. Zinchenko and R.H. Davis (2007). Low-Reynolds-number motion of a deformable drop between two parallel plane walls. Int. J. Multiphase Flow 33, 182-206.

Ho, B.P. and L.G. Leal (1974). Inertial migration of rigid spheres in two-dimensional unidirectional flows. J. Fluid Mech. 65, 365-400.

Janssen, P.J.A. and P.D. Anderson (2008). A boundaryintegral model for drop deformation between two parallel plates with non-unit viscosity ratio drops. J. Computational Physics. 227, 8807-8819. 
Jeffery, R.C. and J.R.A. Pearson (1965). Particle motion in laminar vertical tube flow. J. Fluid Mech. 22, 721 .

Kang, Q., D. Zhang and S. Chen (2005). Displacement of a three-dimensional immiscible droplet in a duct. J. Fluid Mech. 545, 41-66.

Karnis, A., H.L. Goldsmith and S.G. Mason (1963). Axial migration of particles in Poiseuille flow. Nature 200, 159-160.

Karnis, A., H.L. Goldsmith and S.G. Mason (1966). The flow of suspensions through tubes, Inertial effects. Can. J. Chem. Engng. 44, 181-193.

Kowalewski, T.A. (1984). Concentration and velocity measurement in the flow of droplet suspensions through a tube. Experiments in Fluids 2, 213-219.

Liu, S., P.J. Fryer and J.P. Pain (1999). Influence of particle-specific gravity and particle shape on the averaged axial velocity of nearly neutrally buoyant particles in horizontal pipes. Canadian J. Chem. Eng. 77(6), 1083-1089.

Martinez, M.J. and K.S. Udell (1990). Axisymmetric creeping motion of drops though circular tubes. $J$. Fluid Mech. 210, 565-591.

Mortazavi, S.S. and G. Tryggvason (2000). A numerical study of the motion of drops in poiseuille flow, part1: Lateral migration of one drop. J. Fluid Mech. 411, 325-350.

Nourbakhsh, A. and S. Mortazavi (2010). A threedimensional study of the motion of a drop in plane Poiseuille flow at finite Reynolds numbers. (Accepted for publication in Iranian J. Science and Technology, Transaction B: Engineering 34(B2)).

Oliver, D.R. (1962). Influence of particle rotation on radial migration in the Poiseuille flow of suspensions. Nature 194, 1269-1271.

Raiskinmäki, P., A. Koponen, J. Merikoski and J. Timonen (2000). Spreading dynamics of threedimensional droplets by the lattice-Boltzmann method. Computational Materials Science 18, 712.

Ryskin, G. and Leal, L. G. (1984). Numerical solution of free-boundary problems in fluid mechanics, Part 3. Bubble deformation in an axisymmetric straining flow. J. Fluid Mech, Vol. 148, pp. 37-43.

Schleizer, A. D. and Bonnecaze, R. T. (1999). Displacement of a two-dimensional immiscible droplet adhering to a wall in shear and pressuredriven flows. J. Fluid Mech, Vol. 383, pp. 29-54.

Schonberg, J. A. and Hinch, E. J. (1989). Inertial migration of a sphere in poiseuille flow. J. Fluid Mech, Vol. 203, pp. 517-524.
Segre, G. and Silberberg, A. (1962a). Behaviour of macroscopic rigid spheres in poiseuille flow, part1. determination of local concentration by statistical analysis of particle passages through crossed light beams. J. Fluid Mech, Vol. 14, pp. 115-135.

Segre, G. and Silberberg, A. (1962b). Behaviour of macroscopic rigid spheres in poiseuille flow, part2. experimental results and interpretation. J. Fluid Mech, Vol. 14, pp. 136.

Staben, M. E., Galvin, K. P, and Davis, R. H. (2006). Low-Reynolds-number motion of a heavy sphere between two parallel plane walls. Chem. Eng. Sci, Vol. 61, No. 6, pp. 1932-1945.

Taylor, G. I. (1934). The deformation of emulsions in definable fields of flow. Proc. R. Soc. London Ser. A. Vol. 146, pp. 501.

Tryggvason, G., Bunner, B., Esmaeeli, A., Juric, D., AlRawahi, N., Tauber, W., Han, J., Nas, S, and Jan, Y. J. (2001). A front-tracking method for the Computations of Multiphase Flow. J. Computational Physics, Vol. 169, pp. 708-759.

Tsai, T. M. and Miksis, M. J. (1994). Dynamics of a drop in a constricted capillary tube. J. Fluid Mech, Vol. 274, pp. 197-217.

Unverdi, S. O. and Tryggvason, G. (1992a). A fronttracking method for viscous incompressible multifluid flows. J. Comput. Phys, Vol. 100, pp. 25-82.

Unverdi, S. O. and Tryggvason, G. (1992b). Computations of multi-fluid flows. Physics, Vol. 60(D), pp. 70-83.

Vasseur, P. and Cox, R. G. (1976). The lateral migration of a spherical particle in twodimensional shear flows. J. Fluid Mech, Vol. 78, pp. 385 .

Walid, A and Ahmed, O. (2010). Buoyancy induced heat transfer and fluid flow inside a prismatic cavity. To be published in J. Applied Fluid Mech. Vol. 3, No. 2.

Yang, B. H., Wang, J., Joseph D. D., Hu, H. H., Pan, T. W, and Glowinski, R. (2005). Migration of a sphere in tube flow. J. Fluid Mech, Vol. 540, pp. 109-131. 